\title{
GNMT: a multifaceted suppressor of hepatocarcinogenesis
}

\author{
Maria M. SImile, Claudio F. Feo, Diego F. Calvisi, Rosa M. Pascale, Francesco Feo \\ Department of Medical, Surgical and Experimental Sciences, University of Sassari, Sassari 07100, Italy.
}

Correspondence to: Prof. Rosa M. Pascale, Department of Medical, Surgical and Experimental Sciences, University of Sassari, Via P Manzella, 4 07100, Sassari 07100, Italy. E-mail: patsper@uniss.it

How to cite this article: SImile MM, Feo CF, Calvisi DF, Pascale RM, Feo F. GNMT: a multifaceted suppressor of hepatocarcinogenesis. Hepatoma Res 2021;7:35. https://dx.doi.org/10.20517/2394-5079.2020.162

Received: 18 Dec 2020 First Decision: 19 Jan 2021 Revised: 28 Jan 2021 Accepted: 19 Feb 2021 Published: 8 May 2021

Academic Editors: Orlando Musso, Giuliano Ramadori Copy Editor: Xi-Jun Chen Production Editor: Xi-Jun Chen

\begin{abstract}
Glycine N-methyltransferase (GNMT) exerts a pivotal role in the methionine cycle and, consequently, contributes to the control of methylation reactions, and purine and pyrimidine synthesis. Numerous observations indicate that GNMT is a tumor suppressor gene, but the molecular mechanisms of its suppressive action have only been partially unraveled to date. Present knowledge indicates that GNMT acts through both epigenetic and genetic mechanisms. Among them are the decrease of AKT signaling through the inhibition of the RAPTOR/mTOR complex and the interaction of GNMT with the PTEN inhibitor, PREX2. Furthermore, GNMT is a polycyclic aromatic hydrocarbon-binding protein and a mediator of the induction, by polycyclic hydrocarbons of the cytochrome P4501A1 gene, whose polymorphism is involved in favoring different types of cancers. Finally, GNMT suppresses the expression of the transcription factor NRF2, whose overexpression is associated with HCC development. These findings suggest a multifaceted suppressor mechanism of the GNMT gene.
\end{abstract}

Keywords: GNMT, hepatocarcinogenesis, methionine cycle

\section{INTRODUCTION}

Accumulating evidence indicates that glycine N-methyltransferase (GNMT) plays a fundamental role in liver carcinogenesis. In GNMT-KO mice, with the deletion of exons 1-5 of the GNMT gene, HCC grows at the age 14-24 months of age, especially in female mice ${ }^{[1]}$. At the beginning of HCC growth in these mice, Wnt signaling, DNA hypomethylation, overexpression of DNA methyltransferases 1 and $3 \mathrm{~b}$, and activation 
of Gadd45a, Pak1, Mapk3, and Dusp3 genes of the MAPK signaling were found ${ }^{[1]}$. With the deletion of GNMT exon 1 in male mice, HCCs developed at the 8 months age ${ }^{[2]}$. Furthermore, it was found that in AflatoxinB(1)-treated GNMT-KO mice, Cyp1a2, Cyp3a44, Cyp2d22, Gsta4 and Abcasa genes, involved in detoxification, were downregulated ${ }^{[3]}$. Consequently, these mice were highly susceptible to HCC induced by AflatoxinB(1). All these observations indicate that GNMT overexpression confers resistance to liver cancer development.

\section{THE METHIONINE CYCLE}

GNMT is an important component of the "methionine cycle" and, as such, plays a fundamental role in cell physiology ${ }^{[4,5]}$. In this cycle, methionine adenosyltransferases, MATI/III and MATII, convert methionine to S-adenosylmethionine (SAM). The latter has a central role in cell growth, methylation reactions, and glutathione synthesis [Figure 1]. The decarboxylation of SAM by a specific decarboxylase is implicated in the synthesis of polyamines. An end product of this synthesis, 5-methylthioadenosine, is involved in methionine re-synthesis via the "salvage pathway" ${ }^{[5]}$. Various methyltransferases including GNMT are involved in methylation reactions that transform SAM to S-adenosylhomocysteine (SAH). The latter is converted to homocysteine by S-adenosylhomocysteine hydroxylase (SAHH). Homocysteine is transformed by a specific synthetase to cystathionine, a precursor of reduced glutathione. Alternatively, homocysteine may be involved in the re-synthesis of methionine. This may be coupled to the transformation of phosphatidylcholine to phosphatidylethanolamine, operated by phosphatidylethanolamine methyltransferase (PEMT) in the Bremer pathway ${ }^{[6]}$. Phosphatidylcholine is transformed to choline, which is converted to betaine. This is joined to the synthesis of methionine from homocysteine (HCY) to methionine catalyzed by betaine homocysteine methyltransferase (BHMT). The transformation of HCY to methionine may also be associated with the folate cycle $\mathrm{e}^{[7]}$. In this cycle, the methyltetrahydrofolate reductase catalyzes the conversion of tetrahydrofolate (THF) to MeTHF (5,10-methylenetetrahydrofolate), followed by the synthesis of MTHF (5-methyltetrahydrofolate), catalyzed by 5,10-methylene-tetrahydrofolate reductase, that is transformed to methionine by 5 -methyltetrahydrofolate homocysteine methyltransferase [Figure 1].

GNMT catalyzes the methylation by SAM of glycine that is transformed to dimethylglycine (sarcosine). The re-synthesis of glycine catalyzed by sarcosine dehydrogenase (SARDH) is coupled to the transformation of THF into MeTHF [Figure 1]. GNMT binds to MTHF and in turn, is inhibited by MTHF ${ }^{[8]}$. Thus, GNMT induces MTHF retention, is involved in homocysteine generation, and allows MTHF-dependent remethylation of homocysteine, thus contributing to the regulation of methyl group metabolism ${ }^{[8-10]}$.

The methionine and folate cycles undergo multiple interactions with cell metabolism [Figure 2]. SAM "long range interactions" involve the stimulation of GSH synthesis from HCY and the inhibition of BHMT ${ }^{[1-13]}$ and MTHFR $^{[14,15]}$; and therefore to methionine re-synthesis as well as of purine and deoxythymidilate synthesis. In addition, GNMT regulates the SAM/SAH ratio and SAM-dependent methyl transfer reactions. The Km value of GNMT for SAM is relatively high, and GNMT is poorly inhibited by SAH because its Ki value for SAH $(35-80 \mu \mathrm{M})$ is higher than that for other SAM-dependent methyltransferases that are highly inhibited by $\mathrm{SAH}^{[8,13]}$. Thus, GNMT is active at SAM and SAH physiological levels $(0.1-0.2 \mu \mathrm{mol} / \mathrm{g}$ and $0.02-$ $0.06 \mu \mathrm{mol} /$ of the liver, respectively). Its activity may influence SAM/SAH ratio and the activity of other methyltransferases. Besides, GNMT protein binds folate and is inhibited by MTHF $^{[8-11,16]}$. The inhibition of MTHFR by SAM causes the decrease of free MTHF and is followed by the dissociation of GNMT-MTHF complex ${ }^{[13,16]}$. The rise of free GNMT avoids excessive SAM rise. On the contrary, due to the decrease in SAM concentration, MTHFR inhibition is released, MTHF availability rises, and the free GNMT falls. Therefore, by increasing the cellular folate level and thus the MTHFR-dependent homocysteine re- 


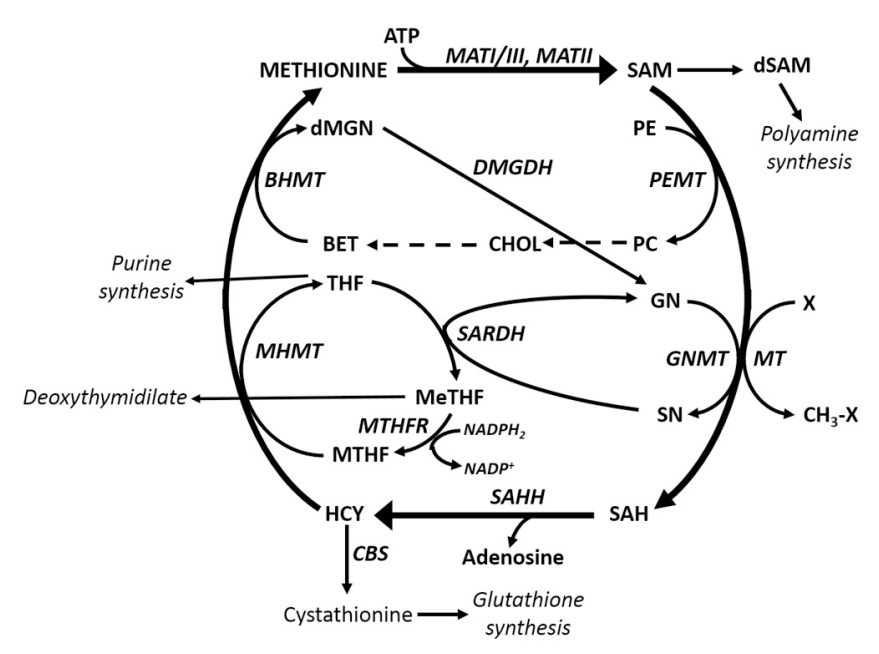

Figure 1. Methionine and folate cycles. Substrates: BET: Betaine; CHOL: choline; dMGN: di-methylglycine; dSAM: decarboxylated Sadenosylmethionine; GN: glycine; HCY: homocysteine; MeTHF: 5,10-methylenetetrahydrofolate; MHMT: methyltetrahydrofolate homocysteine methyltransferase; PC: phosphatidylcholine; PE: phosphatidylethanolamine; SAH: S-adenosylhomocysteine; SAM: Sadenosylmethionine; SN: sarcosine; THF: tetrahydrofolate. Enzymes: BHMT: Betaine homocysteine methyltransferase; CBS: cystathionine beta-synthetase; GNMT: glycine methyltransferase; MATI/III: methionine adenosyltransferase I/III; MATII: methionine adenosyltransferase II; MHMT: methyltetrahydrofolate homocysteine methyltransferase; MT: methyltransferases; MTHFR: 5methyltetrahydrofolate reductase; ODC: ornithine decarbosylase; PEMT: phosphoethanolamine methyltransferase; SAHH: Sadenosylhomocyteine hydroxylase; SNDH: sarcosine dehydrogenase; SARDH: sarcosine dehydrogenase.

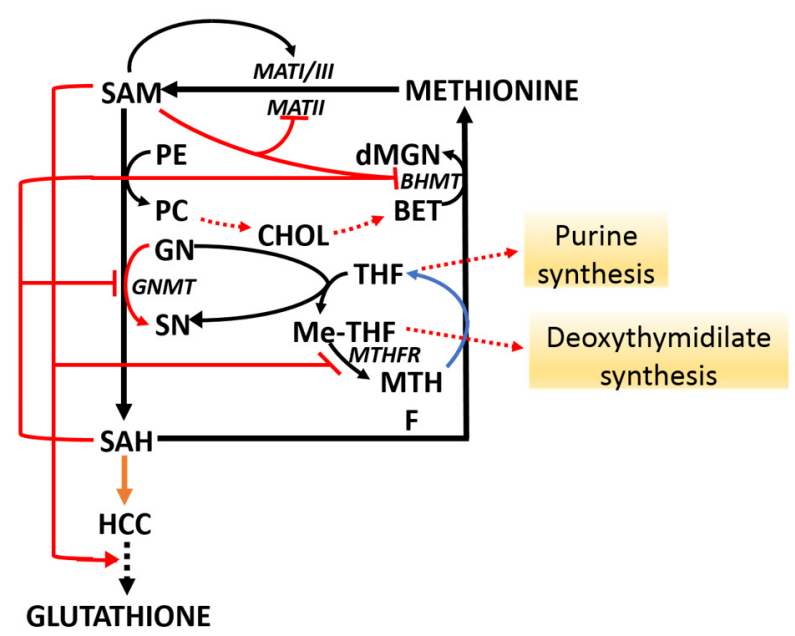

Figure 2. SAM long-range interactions. BET: betaine; BHMT: betaine homocysteine methyltransferase; CHOL: choline; dMGN: dimethylglycine; HCY: homocysteine; MATI/III: methionine adenosyltransferase I/III; MATII: methionine adenosyltransferase II; dMGN: di-methylglycine; GN: glycine; GNMT: glycine methyltransferase; MHMT: methyltetrahydrofolate homocysteine methyltransferase; Me-THF: 5,10-methylenetetrahydrofolate; PC: phosphatidylcholine; PE: phosphatidylethanolamine; SAH: Sadenosylhomocysteine; SAM: S-adenosylmethionine; SN: sarcosine. Red arrows indicate activation; red blunt arrows indicate inhibition.

methylation, GNMT acts as a "salvage pathway".

\section{THE METHIONINE CYCLE DURING LIVER INJURY}

Liver injury from ethanol ${ }^{[17,18]}$, acetaminophen ${ }^{[19]}, \mathrm{CCl}^{[20,21]}$, and galactosamine ${ }^{[22]}$ causes a decrease in SAM content and MAT1A:MAT2A ratio (MAT1A/MAT2A switch) ${ }^{[23]}$. In Mat1A-KO mice at 8 months ${ }^{[24]}$, such decrease induces chronic SAM deficiency that is not compensated by MAT2A induction and is associated with macrovesicular steatosis in up to $50 \%$ of hepatocytes, mononuclear cell infiltration in the periportal 
area, and the development of HCC at 18 months. After the interruption of carcinogen administration to rats, the development of HCCs is associated with a decrease in SAM content and SAM/SAH ratio in dysplastic nodules (DN) and $\mathrm{HCC}^{[5]}$. Low SAM and SAM/SAH ratio are also present in human $\mathrm{HCC}^{[5]}$. The administration of SAM in rats and humans has been shown to decrease liver fibrosis ${ }^{[20,21]}$ and prevent the development of HCCs in rats and mice ${ }^{[24-29]}$.

The decrease in MAT1A expression in human cancer and rat liver carcinogenesis depends on CCGG sequence methylation in MAT1A gene promoter ${ }^{[30-33]}$. In contrast, the promoter of MAT2A gene, hypermethylated and slightly expressed normal liver, is hypomethylated and transcriptionally upregulated in $\mathrm{HCC}^{[32,33]}$. The methylation of MAT1A promoter is also present in HepG2 and Huh7 human liver cancer cell lines. Interestingly, the treatment of HepG2 cells with the demethylating compound 5-aza-2'deoxycytidine or with trichostatin, an histone deacetylase inhibitor, induces a rise of MAT1A expression. This suggests the role of DNA methylation and histone deacetylation in MAT1A silencing ${ }^{[33]}$. In Huh7 cells, the inhibition of MAT1A gene expression is associated with the methylation of CCGG sequence at +10 and +80 of the coding region ${ }^{[31]}$. In contrast, the overexpression of MAT2A gene in human HCC depends on the hypomethylation of CCGG sequences in the $\operatorname{promoter}^{[32,33]}$.

Interestingly, in human HCC with poorer prognosis (HCCP), higher MAT1A:MAT2A switch, CpG methylation, and histone $\mathrm{H} 4$ acetylation were found in human HCC with a better prognosis (HCCB ${ }^{[33]}$. Also, Mat1A/Mat2A switch, CpG hypermethylation, and deacetylation of Mat1A promoter, associated with CpG hypomethylation and histone $\mathrm{H} 4$ acetylation of Mat2A promoter occurred in HCCP of Fisher 344 (F344) rats, genetically susceptible to hepatocarcinogenesis. These alterations were not found in slowly growing HCC of the genetically resistant Brown Norway (BN) rats, indicating their genetic susceptibility to liver cancer ${ }^{[33]}$. Post-transcriptional mechanisms, such as RBPs (RNA binding proteins) AUF1 and HuR are also implicated in MAT1A/MAT2A switch: AUF1 elevates mRNA decay, while HuR binding to AU-rich elements induces mRNA stabilization ${ }^{[34-36]}$. In fetal rat liver, Mat2A and HuR mRNAs are predominantly present. A sharp decrease in the HuR-MAT2A mRNA complexes and an increase in the binding of methylHuR to MAT2A mRNA occur during liver development. This is associated with a reduction in the levels of AUF1-MAT1A mRNA complexes, which correlate with increased MAT1A mRNA. These findings suggest that a balance between methyl-HuR, HuR, and AUF1 regulates the levels of MAT2A and MAT1A mRNAs during liver differentiation ${ }^{[37]}$.

The rise of AUF1 in F344 rats and human HCCs is associated with an intense rise of MAT1A-AUF1 and MAT2A-HuR interactions ${ }^{[33]}$. miRNAs may also contribute to the MAT1A/MAT2A switch. The individual decreases of miR-664, miR-485-3p, and miR-495 in Hep3B and HepG2 cells induce MAT1A expression, while the overexpression of these miRNAs in Hep3B cells to induce tumorigenesis in nude mice is inhibited and increased by their knockdown ${ }^{[3]}$. Furthermore, miR-203 is shown to inhibit MAT2A and MAT2B expression and growth, while it increases apoptosis of HepG2 and Huh7 liver cancer cells ${ }^{[38]}$.

\section{GNMT DURING LIVER INJURY}

GNMT protein and its enzymatic activity are present in the liver ${ }^{[39]}$. Low GNMT expression was found in cirrhotic liver, HCC associated with hepatitis C virus and alcohol abuse, and in human HCC cell lines ${ }^{[00-43]}$. Steatohepatitis fibrosis, cirrhosis, and HCC develop in GNMT deficient mice whereas the GNMT inducer 1,2,3,4,6-penta-O-galloyl- $\beta$-d-glucopyranoside (PGG) inhibits HCC development in vitro and in vivo ${ }^{[44]}$. Interestingly, fast-growing HCCs are characterized by the complete absence of GNMT that may be present in low levels in slow-growing HCC. This suggests an influence of GNMT on tumor progression ${ }^{[45]}$. In pancreatic adenocarcinoma, GNMT gene is not expressed because of the methylation of its promoter ${ }^{[46]}$. 
Analogous GNMT hypermethylation is present in some HCC cell lines and $20 \%$ of primary HCCs ${ }^{[47]}$. Interestingly, GNMT binds some carcinogenic compounds, including aromatic hydrocarbons and aflatoxins. This prevents the formation of DNA adducts and carcinogen cytotoxicity ${ }^{[48-50]}$. Studies of GNMT, performed in HCC of Taiwanese men, indicated that GNMT may be a tumor susceptibility gene for this tumor $^{[51]}$. Furthermore, the presence of GNMT was not detected in SK-Hep1, Hep 3B, Huh-7, and HA22T human HCC cell lines. The investigation of a Taiwanese liver cDNA library allowed the isolation of the clone-9-1-2 full-length GNMT cDNA. This clone, compared with the GNMT cDNA sequence, exhibited 4 nucleotide differences, causing the change of an amino-acid. Immunohistochemical staining with the rabbit anti-recombinant GNMT serum showed that GNMT protein almost completely disappeared in cancer cells $^{[51]}$. Other researches ${ }^{[52,53]}$ revealed the GNMT short tandem repeat polymorphism (STRP1) and single nucleotide polymorphism were not associated with a rise in the risk of prostate cancer in Taiwanese men of European descendants ${ }^{[53]}$. In prostate cancer, it was identified as an androgen-responsive element in the first exon of the GNMT gene, binding androgen receptor in vitro and in vivo ${ }^{[54,5]}$. This indicates that androgen is an important regulator of GNMT gene. Furthermore, GNMT-KO mice, lacking exons 1 of the GNMT gene, develop HCC. This was associated with hypermethioninemia and elevated levels of serum aminotransferase and liver $\mathrm{SAM}^{[5,56]}$. In about $60 \%$ of these mice, high glycogen storage in the liver was detected and HCC prevalently developed in female mice at 14-24 months of age ${ }^{[56]}$.

An important contribution to understanding the role of GNMT liver carcinogenesis was the demonstration in GNMT-KO mice of 7- and 35-fold rises in free methionine and SAM, respectively, associated with a 3fold decrease of SAH level ${ }^{[57]}$. At the early stages of HCC development, global DNA hypomethylation and aberrant expression of DNA methyltransferases 1 and $3 b$ were observed ${ }^{[1]}$. In a male GNMT-KO model with disrupted GNMT exon 1, high serum aminotransferases, methionine, and SAM levels were associated with liver steatosis, fibrosis, and HCC development at the age of 8 months $^{[2]}$. Tumors in these mice were characterized by the activation of Ras and Jak/Stat (Janus kinase/signal transducer and activator of transcription) pathways, and suppression of Ras inhibitors RASSF 1 and 4, Jak/Stat inhibitors SOCS 1-3, and cytokine-inducible SH2-protein ${ }^{[2]}$. Hypermethylation of Rassf1 and Socs2 promoters and binding of trimethylated lysine 27 of histone 3 to these 2 genes occurred in HCCs from GNMT-KO mice ${ }^{[2]}$. This indicates that the presence of aberrant methylation of DNA and histones caused the epigenetic modulation of carcinogenic pathways. Some interactions also occur between GNMT and LKB1/AMPK signaling; indeed, HCC cells from GNMT-KO mice lacking exon 1 showed, like human HCCs, overexpression of the Ras gene that mediated the hyper-activation of Lkb1 with consequent AMPK activation ${ }^{[3]}$. This requires Erk2 and p9orsk activity and Rasgrp3 expression. Interestingly, in HCCP, reduced levels of GNMT, phosphorylation of AMPK $\alpha$ at Thr172, and overexpression of RAS, LKB1, and RASGRP3 have been reported $^{[57]}$.

\section{GNMT EXPRESSION IN TRANSCRIPTOMIC CLASSIFICATIONS OF HCC}

Human HCCs have been classified based on metabolic features ${ }^{[58]}$ in tumors with better (HCCB) and poorer (HCCP) prognosis ${ }^{[59]}$. Interestingly, in HCCP, a higher MAT1A:MAT2A switch, CpG methylation, and histone $\mathrm{H} 4$ acetylation occur, with respect to $\mathrm{HCCB}^{[33]}$. Analogous alterations were found in $\mathrm{HCC}$ of $\mathrm{F} 344$ rats, genetically susceptible to hepatocarcinogenesis. In contrast, these alterations were absent in slowly growing $\mathrm{HCC}$ of the genetically resistant $\mathrm{BN}$ rats ${ }^{[33]}$. Interestingly, it was observed that genes involved in methionine metabolism, such as BHMT and GNMT, are also involved in the resistance to liver cancer ${ }^{[59]}$. A gene expression signature with the highest expression of proliferation-related CTGF, $c-M Y C$, and PCNA genes, was associated with the lowest expression of the onco-suppressors BHMT, DMBT1, DUSP1, GADD45g and GNMT genes, in HCCP ${ }^{[59]}$. An overexpression of the onco-suppressors Bhmt, Dmbt1, Dusp1, Gadd45g, GNMT, Napsa, Pp2ca, and Ptpn13 was found in HCCs of BNA rats ${ }^{[59,60]}$. In light of these findings, 
it is interesting that genes involved in methionine metabolism, such as BHMT and GNMT, are also involved in the resistance to liver cancer ${ }^{[59]}$. The mechanisms by which GNMT participates in tumor prevention/suppression in humans are not completely clear. It has been shown ${ }^{[1]}$ that GNMT supports methylene-folate dependent pyrimidine synthesis and formyl-folate dependent purine synthesis [Figure 2]. It also minimizes uracil incorporation into DNA because of folate depletion and translocates into the nucleus during prolonged folate depletion. According to these findings, loss of GNMT impairs nucleotide biosynthesis, whereas GNMT overexpression enhances nucleotide biosynthesis and improves DNA integrity by reducing uracil misincorporation in DNA both in vitro and in vivo ${ }^{[1]}$.

\section{GNMT AND LIVER CANCER SUPPRESSION}

Studies on GNMT-KO mice indicate that GNMT is a suppressor gene and confirm previous findings suggesting that down-regulation of GNMT gene expression may be involved in the pathogenesis of liver cancer. Low levels of GNMT activity were detected in fetal rabbit liver about 20 days post-fertilization, it increased to higher levels after birth, reaching maximum in the adult liver ${ }^{[45]}$. In fast-growing rat HCCs, GNMT activity is absent, but it can be detected in the slower-growing HCCs at lower levels than in normal adult rat liver, suggesting a role of GNMT in HCC progression ${ }^{[42]}$. Furthermore, GNMT could be involved in stress responses induced by low-dose irradiation of mouse liver ${ }^{[59]}$. GNMT also regulates genes implicated in the detoxification and anti-oxidation pathways, and Benz(a)pyrene carcinogenicity ${ }^{[60]}$. It is also an essential regulator of Complex II activity in the mitochondrial electron transport chain ${ }^{[6]]}$.

GNMT-KO has allowed the study of liver progenitor cells and their role in hepatocarcinogenesis. E. Farber first hypothesized that progenitor cells resistant to liver injury proliferate to form preneoplastic dysplastic nodules and HCCs by the "resistant hepatocyte protocol" ${ }^{62]}$. The GNMT-KO mice model allows a detailed study of these cells. In these mice livers, hyperplasia of progenitor OV-6 positive cells occurs at 8 months, a process that increases at 18 months when HCC develops and expresses Fat10, the marker for progenitor liver cells ${ }^{[2]}$. In an analogous liver cancer model, in mice that are fed diethyl-1,4-dihydro-2,4,6-trimethyl3,5-pyridine decarboxylate (DDC), stem cell/progenitor cell (SCP) are upregulated as indicated by microarrays, and are prevented by $\mathrm{SAM}^{[63]}$.

The mechanisms of tumor suppression mediated by GNMT are complex and probably not completely known. The protein mTOR (mechanistic target of rapamycin) controls cell growth and translation by the assembly of multi-protein signaling complexes, the best described of which is mTORC1 (mechanistic target of rapamycin complex 1) ${ }^{[64]}$. In this complex, mTOR is associated with RAPTOR (regulatory associated protein of mTOR), LST8, and PRAS40 proteins ${ }^{[65]}$. The increase in mTORC 1 activates S6K1/2 proteins and represses $4 \mathrm{E}-\mathrm{BPs}{ }^{[6]}$. RAPTOR over-expression activates AKT via the inhibition of the negative feedback loop from S6K1 to ISR (insulin receptor substrate) ${ }^{[65]}$. The RAPTOR/mTOR complex, overexpressed in HCC, interacts with GNMT ${ }^{[65]}$ [Figure 3]. A decrease in mTORC1 signaling, induced by GNMT overexpression, inhibits G2/M cell cycle progression, and Huh-7 cell proliferation ${ }^{[65]}$.

PREX2 (phosphatidylinositol 3,4,5-trisphosphatedependent Rac exchanger 2) regulates the small guanosine triphosphatase, Rac, and induces the growth and migration of HCC cells by the PTEN-AKT signaling. Moreover, PREX2 overexpression in HCC inhibits the activity of PTEN (phosphatase and tensin homolog), which regulates HCC cell invasiveness ${ }^{[6]]}$. The inactivation of PTEN by PREX2, in turn, inactivates AKT. The opposite occurs by the knockdown of PREX2 expression ${ }^{[6]]}$. The inhibition of AKT signaling is also induced by GNMT interaction with the PTEN inhibitor, PREX ${ }^{[67,68]}$. This interaction enhances the proteasomal degradation of PREX2 by the E3 ubiquitin ligase, HectH9 [Figure 4]. Prex2 protein overexpression, in association with Akt activation, occurs in the liver of GNMT-KO mice ${ }^{[6]]}$. Elevated levels 


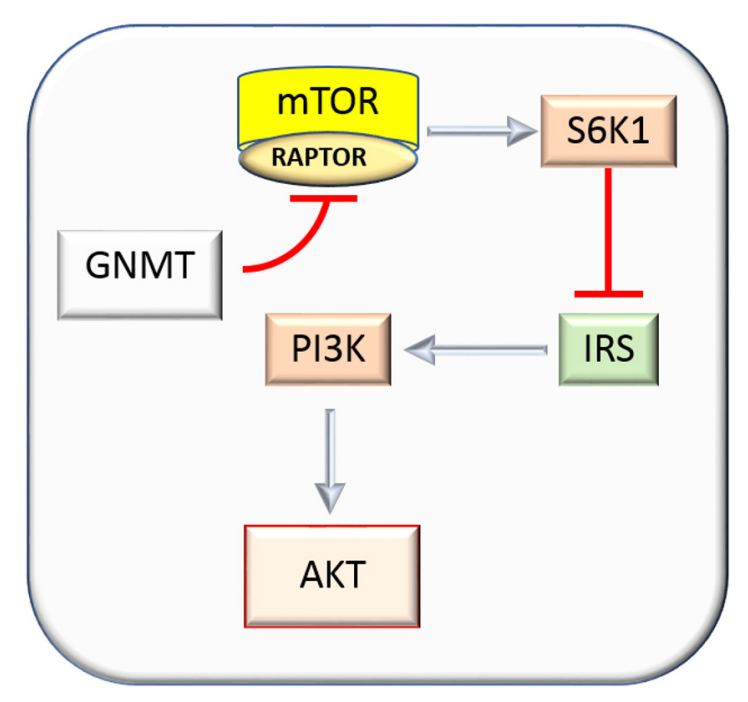

Figure 3. Mechanisms of inhibition of hepatocellular carcinoma growth by glycine methyltransferase. The inhibition of the RAPTOR/mTOR complex, impedes S6K1 activation and the feedback negative effect of S6K1 on the PI3K/AKT axis mediated by IRS. GNMT: glycine N-methyltransferase; mTOR: mechanistic target of rapamycin; IRS: insulin response element; PI3K: phosphoinositide-3kinase.

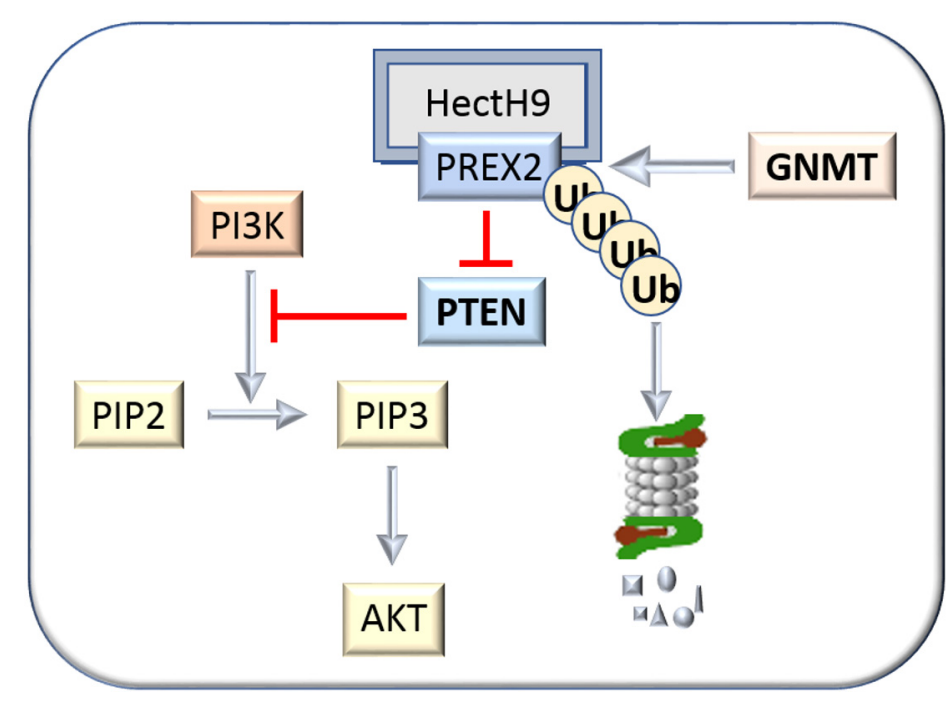

Figure 4. Inhibition of PREX2proteasomal degradation by GNMT. AKT: Murine thymoma viral oncogene homolog; GNMT: glycine Nmethyltransferase; HectH9: E3 ubiquitin ligase; PI3K: phosphoinositide-3-kinase; PIP2: phosphorylating phosphatidylinositol-4,5bisphosphatde; PIP3: phosphatidylinositol-3,4,5-triphosphate; PREX2: phosphatidylinositol 3,4,5-trisphosphatedependent Rac exchanger 2 .

of PREX2 were also found in human HCC and associated with decreased survival. In these HCCs, PREX2 $m R N A$ expression is not decreased, indicating that a post-translational alteration of PREX2 expression occurs $^{[67]}$.

The above mechanisms of inhibition of the suppressive effects of GNMT are epigenetic. However, the transport of rat GNMT into liver nuclei, where it interacts with chromatin, has also been observed ${ }^{[68,69]}$. Nuclear GNMT has different and sometimes contradictory effects. The GNMT nuclear localization induces apoptosis, independently of its catalytic activity or folate binding capacity ${ }^{[70]}$. The anti-proliferative effect of 
GNMT is partially reversed by the pan-caspase inhibitor, zVAD-fmk ${ }^{[69]}$.

GNMT translocation into the nucleus supports pyrimidine and purine synthesis, and lessens uracil incorporation into DNA in cells with folate depletion ${ }^{[70,71]}$. Its loss impairs nucleotide biosynthesis ${ }^{[72]}$. GNMT suppressor activity also relates to the regulation of cytochrome $P 450-1 A 1$ gene expression ${ }^{[71,72]}$. The polymorphism of this gene increases the susceptibility to breast ${ }^{[73]}$, cervical ${ }^{[73]}$, and lung ${ }^{[74]}$ cancers. The tobacco-derived polycyclic aromatics hydrocarbons are implicated in the risk of HCC in chronic HBV carriers, and CYP1A1 polymorphism is an important modulator of the hepatocarcinogenic effect of these carcinogens ${ }^{[75]}$.

Recent research has shown that NRF2 overexpression is implicated in HCC development ${ }^{[76]}$. NRF2 translocation to the nucleus induces the dimerization with sMAF proteins ${ }^{[77]}$. The complex NRF2/sMAF binds antioxidant response element (ARE), activating the transcription of target genes [Figure 5]. The transcription factor, $\mathrm{NRF} 2$, is a main regulator of the antioxidative and detoxification responses and propels the progression of cancer, the formation of metastases, and the resistance to therapy ${ }^{[78]}$. Interestingly, nuclear GNMT interacts with the promoter region of the genes encoding NRF2 ${ }^{[79]}$.

In the cirrhotic liver of cholestatic patients and animal models of liver cholestasis and cirrhosis, miR-873-5p is inversely correlated with GNMT expression; high circulating miR-873-5 is present in cholestatic patient $^{[80]}$. The treatment with anti-miR-873-5p in Mdr2-/-mice with bile duct ligation recovers GNMT levels and improves the inflammation and fibrosis by counteracting hepatocyte apoptosis and cholangiocyte proliferation ${ }^{[80]}$. Furthermore, in patients with non-alcoholic fatty liver disease (NAFLD) and a preclinical murine non-alcoholic steatohepatitis (NASH) model, miR-873-5p controls GNMT expression of hepatocytes, leading to the disruption of mitochondrial functionality ${ }^{[81]}$. GNMT is an essential regulator of Complex II activity in the electron transport chain of mitochondria ${ }^{[81]}$. The upregulation of miR-873-5p in the liver of NAFLD/NASH patients is correlated with GNMT depletion. Importantly, NASH therapies based on anti-miR-873-5p, sort out lipid accumulation, inflammation, and fibrosis by enhancing fatty acid $\beta$ -oxidation in mitochondria ${ }^{[8]}$. GNMT participates in the regulation of metabolic pathways and mitochondrial functionality through the regulation of Complex II activity in the electron transport chain ${ }^{[81]}$.

The observations that GNMT knockout mice developed HCC and GNMT prevented aflatoxin-induced carcinogenicity and inhibited HCC cell proliferation, motivated the research on GNMT inducers for HCC therapy. The extract of the active component of Paeonia lactiflora Pall, PGG, was identified as a GNMT inducer. PGG treatment sensitized Huh7 cells to sorafenib treatment ${ }^{[82]}$. These findings explain why PGG could have therapeutic potential for the treatment of $\mathrm{HCC}^{[44]}$. However, at present, cancer therapy that stems from alterations in the methionine cycle is based on the inhibitory effects of SAM. Different studies have documented the decrease of hepatic SAM content during acute and chronic alcoholism ${ }^{[1,83]}$, and the prevention of fatty liver and GSH decrease by the administration of exogenous $\mathrm{SAM}^{[17]} \cdot \mathrm{Na}^{+}, \mathrm{K}^{+}$-ATPase is an integral plasma membrane enzyme that plays a key role in the physiology and structure of hepatocytes, where it maintains the electrochemical gradients for $\mathrm{Na}^{+}$and $\mathrm{K}^{+}$across the cell membrane. Its activity is inhibited by ethanol that determines a decrease in GSH liver content and steatosis. SAM accelerates the recovery of these parameters after ethanol withdrawal and also protects $\left(\mathrm{Na}^{+}, \mathrm{K}^{+}\right)$ATPase activity and GSH content of isolated hepatocytes from the deleterious effect of ethanol ${ }^{[1784,85]}$. The maintenance of a high GSH level by SAM determines its beneficial effect that indeed is prevented by 1-chloro-2,4-dinitro-benzene, a compound that depletes cell GSH ${ }^{[86]}$. 


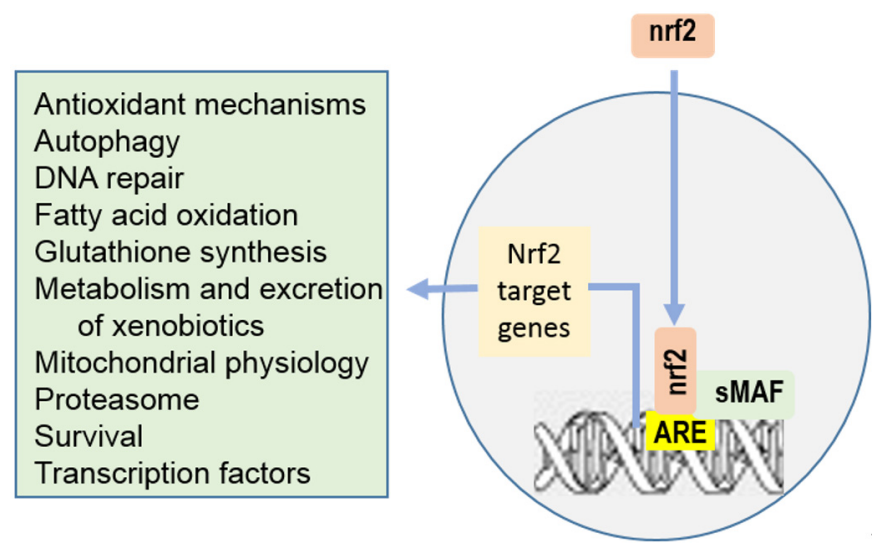

Figure 5. Effects of Nrf2 nuclear translocation. After nuclear translocation, NRF2 dimerizes with sMAF proteins, and together they bind ARE to activate the transcription of NRF2 target genes. Some processes regulated by NRF2 target genes are shown. ARE: Antioxidant response element.

Present knowledge indicates that both epigenetic and genetic mechanisms may be involved in cancer suppression by GNMT. The epigenetic mechanisms implicate the inhibitory effects of GNMT on AKT signaling, which in turn, is linked to its interaction with MTOR and PREX2 $2^{[64-67]}$. Less clear are the genetic mechanisms responsible for the suppressor activity of GNMT. A connection of GNMT with the regulation of $C_{Y P} P_{1} A_{1}$ and $N R F 2$ genes is suggested ${ }^{[71-76]}$, bsut further work is necessary to clarify this aspect of the GNMT suppressor activity.

\section{DECLARATIONS}

\section{Authors' contributions}

Organized and wrote the review: Simile MM, Feo CF, Calvisi DF, Pascale RM, Feo F All Authors contributed equally.

\section{Availability of data and materials}

Not applicable.

\section{Financial support and sponsorship}

This work was supported by Grant: Fondazione di Sardegna: R.M. Pascale, 2017.

\section{Conflicts of interest}

All authors declared that there are no conflicts of interest.

\section{Ethical approval and consent to participate}

Not applicable.

\section{Consent for publication}

Not applicable.

\section{Copyright}

(c) The Author(s) 2021. 


\section{REFERENCES}

1. Liao YJ, Liu SP, Lee CM, et al. Characterization of a glycine N-methyltransferase gene knockout mouse model for hepatocellular carcinoma: Implications of the gender disparity in liver cancer susceptibility. Int J Cancer 2009;124:816-26. DOI PubMed

2. Martínez-Chantar ML, Vázquez-Chantada M, Ariz U, et al. Loss of the glycine N-ethyltransferase gene leads to steatosis and hepatocellular carcinoma in mice. Hepatology 2008;47:1191-9. DOI PubMed PMC

3. Liu SP, Li YS, Lee CM, et al. Higher susceptibility to aflatoxin B1-related hepatocellular carcinoma in glycine N-methyltransferase knockout mice. Int J Cancer 2011:128;511-23. DOI PubMed

4. JD. Methionine metabolism in mammals. J Nutr Biochem 1990;1:228-37. DOI PubMed

5. Pascale RM, Peitta G, Simile MM, Feo F. Alterations of Methionine Metabolism as Potential Targets for the Prevention and Therapy of Hepatocellular Carcinoma. Medicina (Kaunas) 2019;55:296. DOI PubMed PMC

6. Bremer J, Greenberg DM. Biosynthesis of choline in vitro. Biochim Biophys Acta 1960;37:173-5. DOI PubMed

7. Blom HJ, Smulders Y. Overview of homocysteine and folate metabolism. With special references to cardiovascular disease and neural tube defects. J Inherit Metab Dis 2011;34:75-81. DOI PubMed PMC

8. Cook RJ, Wagner C. Glycine N-methyltransferase is a folate binding protein of rat liver cytosol. Proc Natl Acad Sci U S A 1984;81:3631-4. DOI PubMed PMC

9. Wang YC, Wu MT, Lin YJ, Tang FY, Ko HA, et al. Regulation of Folate-Mediated One-Carbon metabolism by GlycineNMethyltransferase (GNMT) and Methylenetetrahydrofolate reductase (MTHFR). J Nutr Sci Vitaminol (Tokyo) 2015;61:S148-50. DOI PubMed

10. Williams KT, Schalinske KL. New insights into the regulation of methyl group and homocysteine metabolism. $J$ Nutr 2007;137:311 4. DOI PubMed

11. Reed MC, Gamble MV, Hall MN, Nijhout HF. Mathematical analysis of the regulation of competing methyltransferases. BMC Syst Biol 2015;9:69. DOI PubMed PMC

12. Finkelstein JD, Martin JJ. Inactivation of betaine-homocysteine methyltransferase by adenosylmethionine and adenosylethionine. Biochem Biophys Res Commun 1984;118:14-9. DOI PubMed

13. Ou X, Yang H, Ramani K, et al. Inhibition of human betaine-homocysteine methyltransferase expression by S-adenosylmethionine and methylthioadenosine. Biochem J 2007;401:87-96. DOI PubMed PMC

14. Kutzbach C, Stokstad ELR. Feedback inhibition in methylenetetrahydrofolate reductase in rat liver by S-adenosylmethionine. Biochim Biophys Acta 1967;139:217-20. DOI PubMed

15. Jencks DA, Matthews RG. Allosteric inhibition of methylenetetrahydrofolate reductase by adenosylmethionine. Effects of adenosylmethionine and NADPH on the equilibrium between active and inactive forms of the enzyme and on the kinetics of approach

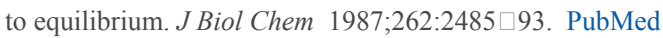

16. Wagner C, Briggs WT, Cook RJ. Inhibition of glycine N-methyltransferase activity by folate derivatives: implications for regulation of methyl group metabolism. Biochem Biophys Res Commun 1985;127:746-52. DOI PubMed

17. Feo F, Pascale R, Garcea R, et al. Effect of the variations of S-adenosyl-L-methionine liver content on fat accumulation and ethanol metabolism in ethanol-intoxicated rats. Toxicol Appl Pharmacol 1986;83:331-41. DOI PubMed

18. Lieber CS, Casini A, DeCarli LM, Kim CI, et al. S-adenosyl-L-ethionine attenuates alcohol-induced liver injury in the baboon. Hepatology 1990;11:165-72. DOI PubMed

19. Stramentinoli G, Pezzoli C, Galli-Kienle M. Protective role of S-adenosyl-methionine against acetaminophen induced mortality and hepatotoxicity in mice. Biochem Pharmacol 1979;28:3567-3571. DOI PubMed

20. Corrales F, Giménez A, Alvarez L, et al. S-adenosylmethionine treatment prevents carbon tetrachloride-induced S-adenosylmethionine synthetase inactivation and attenuates liver injury. Hepatology 1992;16:1022-7. DOI PubMed

21. Simile MM, Banni S, Angioni E, et al. 5-Methylthioadenosine administration prevents lipid peroxidation and fibrogenesis induced in rat liver by carbon-tetrachloride intoxication. J Hepatol 2001;34:386-94. DOI PubMed

22. Stramentinoli G, Gualano M, Ideo G. Protective role of S-adenosylmethionine on liver injury induced by D-galactosamine in rats. Biochem Pharmacol 1978;27:1431-3. DOI PubMed

23. Mato JM, Corrales FJ, Lu SC, Avila MA. S-adenosylmethionine: a control switch that regulates liver function. FASEB J 2002;16:1526. DOI PubMed

24. Lu SC, Mato JM. Role of methionine adenosyltransferase and S-adenosylmethionine in alcohol-associated liver cancer. Alcohol 2005;35:227-34. DOI PubMed

25. Lu SC, Alvarez L, Huang ZZ, et al. Methionine adenosyltransferase 1A knockout mice are predisposed to liver injury and exhibit increased expression of genes involved in proliferation. Proc Natl Acad Sci U S A 2001;98:5560-5. DOI PubMed PMC

26. Pascale RM, Simile MM, Satta G, et al. Comparative effects of L-methionine, S-adenosyl-L-methionine and 5-methylthioadenosine on the growth of preneoplastic lesions and DNA methylation in rat liver during the early stages of hepatocarcinogenesis. Anticancer Res 1991;11:1617-24. PubMed

27. Garcea R, Daino L, Pascale RM, et al. Inhibition of promotion and persistent nodule growth by S-adenosyl-L-methionine in rat liver carcinogenesis: role of remodeling and apoptosis. Cancer Res 1989;49:1850-6. PubMed

28. Pascale RM, Simile MM, De Miglio MR, et al. Chemoprevention by S-adenosyl-L-methionine of rat liver carcinogenesis initiated by 1,2-dimethylhydrazine and promoted by orotic acid. Carcinogenesis 1995;16:427-30. DOI PubMed

29. Lu SC, Ramani K, Ou X, et al. S-adenosylmethionine in the chemoprevention and treatment of hepatocellular carcinoma in a rat model. Hepatology 2009;50:462-471. DOI PubMed PMC 
30. Torres L, Avila MA, Carretero MV, et al. Liver-specific methionine adenosyltransferase MAT1A gene expression is associated with a specific pattern of promoter methylation and histone acetylation:implications for MAT1A silencing during transformation. FASEB $J$ 2000;14:95-102. DOI PubMed

31. Tomasi ML, Li TW, Li M, Mato JM, Lu SC. Inhibition of human methionine adenosyltransferase 1A transcription by coding region methylation. J Cell Physiol 2012;227:1583-91. DOI PubMed PMC

32. Yang H, Huang ZZ, Zeng Z, Chen C, Selby RR, Lu SC. Role of promoter methylation in increased methionine adenosyltransferase 2A expression in human liver cancer. Am J Physiol Gastrointest Liver Physiol 2001;280:G184-90. DOI PubMed

33. Frau M, Tomasi ML, Simile MM, et al. Role of transcriptional and posttranscriptional regulation of methionine adenosyltransferases in liver cancer progression. Hepatology 2012;56:165-75. DOI PubMed

34. Feo F, De Miglio MR, Simile MM, et al. Hepatocellular carcinoma as a complex polygenic disease. Interpretive analysis of recent developments on genetic predisposition. Biochim Biophys Acta 2006;1765:126-47. DOI PubMed

35. Lal A, Mazan-Mamczarz K, Kawai T, Yang X, Martindale JL, Gorospe M. Concurrent versus individual binding of HuR and AUF1 to common labile target mRNAs. EMBO J 2004;23:3092-102. DOI PubMed PMC

36. Kim MY, Hur J, Jeong S. Emerging roles of RNA and RNA-binding protein network in cancer cells. BMB Rep 2009;42:125-130. DOI PubMed

37. Vázquez-Chantada M, Fernández-Ramos D, Embade N, et al. HuR/-methyl-HuR and AUF1 regulate the MAT expressed during liver proliferation, differentiation, and carcinogenesis. Gastroenterology 2010;138:1943-53. DOI PubMed PMC

38. Simile MM, Peitta G, Tomasi ML, et al. MicroRNA-203 impacts on the growth, aggressiveness and prognosis of hepatocellular carcinoma by targeting MAT2A and MAT2B genes. Oncotarget 2019;10:2835-54. DOI PubMed PMC

39. Yang H, Cho ME, Li TW, et al. MicroRNAs regulate methionine adenosyltransferase 1A expression in hepatocellular carcinoma. $J$ Clin Invest 2013;123:285-98. DOI PubMed PMC

40. Yeo EJ, Wagner C. Tissue distribution of glycine N-methyltransferase, a major folate-binding protein of liver. Proc Natl Acad Sci U S A 1994;91:210-4. DOI PubMed PMC

41. Avila MA, Berasain C, Torres L, et al. Reduced mRNA abundance of the main enzymes involved in methionine metabolism in human liver cirrhosis and hepatocellular carcinoma. J Hepatol 2000;33:907-14. DOI PubMed

42. Frau M, Feo F, Pascale RM. Pleiotropic effects of methionine adenosyltransferases deregulation as determinants of liver cancer progression and prognosis. J Hepatol 2013;59:830-41. DOI PubMed

43. Chen YM, Shiu JY, Tzeng SJ, et al. Characterization of glycine-N-methyltransferase-gene expression in human hepatocellular carcinoma. Int J Cancer 1998;75:787-93. DOI PubMed

44. Kant R, Yen CH, Lu CK, Lin YC, Li JH, Chen YM. Identification of 1,2,3,4,6-Penta-O-galloyl- $\beta$-d-glucopyranoside as a Glycine-nmethyltransferase enhancer by high-throughput screening of natural products inhibits hepatocellular carcinoma. Int J Mol Sci 2016;17:669. DOI PubMed PMC

45. Heady JE, Kerr SJ. Alteration of glycine N-methyltransferase activity: fetal, adult, and tumor tissues. Cancer Res 1975;35:640-3. PubMed

46. Botezatu A, Bleotu C, Nastase A, et al. Epigenetic silencing of GNMT gene in pancreatic adenocarcinoma. Cancer Genomics Proteomics 2015;12:21-30. PubMed

47. Huidobro C, Toraño EG, Fernández AF, et al. A DNA methylation signature associated with the epigenetic repression of glycine Nmethyltransferase in human hepatocellular carcinoma. J Mol Med (Berl) 2013;91:939-50. DOI PubMed

48. Bhat R, Bresnick E. Glycine N-methyltransferase is an example of functional diversity. Role as a polycyclic aromatic hydrocarbon binding receptor. J Biol Chem 1997;272:21221-6. DOI PubMed

49. Chen SY, Lin JR, Darbha R, Lin P, Liu TY, Chen YM. Glycine N-methyltransferase tumor susceptibility gene in the benzo(a)pyrene detoxification pathway. Cancer Res 2004;64:3617-23. DOI PubMed

50. Yen CH, Hung JH, Ueng YF, et al. Glycine N-methyltransferase affects the metabolism of aflatoxin B1 and blocks its carcinogenic effect. Toxicol Appl Pharmacol 2009;235:296-304. DOI PubMed

51. Tseng TL, Shih YP, Huang YC, et al. Genotypic and phenotypic characterization of a putative tumor susceptibility gene, GNMT, in liver cancer. Cancer Res 2003;63:647-54. PubMed

52. Huang YC, Lee CM, Chen M, et al. Haplotypes, loss of heterozygosity, and expression levels of glycine N-methyltransferase in prostate cancer. Clin Cancer Res 2007;13:1412-20. DOI PubMed

53. Chen M, Huang YL, Huang YC, et al. Genetic polymorphisms of the glycine N-methyltransferase and prostate cancer risk in the health professionals follow-up study. PLoS One 2014;9:e94683. DOI PubMed PMC

54. Ottaviani S, Brooke GN, O’Hanlon-Brown C, Waxman J, Ali S, Buluwela L. Characterisation of the androgen regulation of glycine Nmethyltransferase in prostate cancer cells. J Mol Endocrinol 2013;51:301-12. DOI PubMed PMC

55. Lee CM, Yen CH, Tzeng TY, et al. Androgen response element of the glycine N-ethyltransferase gene is located in the coding region of its first exon. Biosci Rep 2013;3 3(5):e00070. DOI PubMed PMC

56. Luka Z, Capdevila A. , Mato JM, Wagner C. A glycine N-methyltransferase knockout mouse model for humans with deficiency of this enzyme. Transgenic Res 2006;15:393-7. DOI PubMed PMC

57. Martínez-López N, García-Rodríguez JL, Varela-Rey M, et al. Hepatoma cells from mice deficient in glycine N-methyltransferase have increased RAS signaling and activation of liver kinase B1. Gastroenterology 2012;143:787-98. DOI PubMed PMC

58. Yang C, Huang X, Liu Z, Qin W, Wang C. Metabolism-associated molecular classification of hepatocellular carcinoma. Mol Oncol 2020;14:896-913. DOI PubMed PMC

59. Frau M, Simile MM, Tomasi ML, et al. An expression signature of phenotypic resistance to hepatocellular carcinoma identified by 
cross-species gene expression analysis. Cell Oncol (Dordr) 2012;35:163-73. DOI PubMed PMC

60. Yi L, Li L, Yin J, Hu N, Li G, Ding D. Proteomics analysis of liver tissues from C57BL/6J mice receiving low-dose 137Cs radiation. Environ Sci Pollut Res Int 2016;23:2549-56. DOI PubMed

61. Farber E. Cloncal adaption during carcinogenesis. Biochem Pharmacol 1990;39:1837-46. DOI PubMed

62. Martinez-Chantar ML, Lu SC, Mato JM, et al. The role of stem cells/progenitor cells in liver carcinogenesis in glycine Nmethyltransferase deficient mice. Exp Mol Pathol 2010;88:234-7. DOI PubMed PMC

63. Li J, Bardag-Gorce F, Dedes J, et al. S-adenosylmethionine prevents Mallory Denk body formation in drug-primed mice by inhibiting the epigenetic memory. Hepatology 2008;47:613-24. DOI PubMed PMC

64. Hoeffer CA, Klann E. mTOR signaling: at the crossroads of plasticity, memory and disease. Trends Neurosci 2010;33:67-75. DOI PubMed PMC

65. Yen $\mathrm{CH}, \mathrm{Lu} \mathrm{YC}, \mathrm{Li} \mathrm{CH}$, et al. Functional characterization of glycine $\mathrm{N}$-methyltransferase and its interactive protein DEPDC6/DEPTOR in hepatocellular carcinoma. Mol Med 2012;18:286-96. DOI PubMed PMC

66. Li CH, Yen CH, Chen YF, et al. Characterization of the GNMT HectH9-PREX2 tripartite relationship in the pathogenesis of hepatocellular carcinoma. Int J Cancer 2017;140:2284-97. DOI PubMed

67. He S, Lin J, Yu S, Sun S. Upregulation of PREX2 promotes the proliferation and migration of hepatocellular carcinoma cells via PTEN-AKT signaling. Oncol Lett 2016;11:2223-8. DOI PubMed PMC

68. DebRoy S, Kramarenko II, Ghose S, Oleinik NV, Krupenko SA, Krupenko NI. A novel tumor suppressor function of glycine Nmethyltransferase is independent of its catalytic activity but requires nuclear localization. PLoS One 2013;8:e70062. DOI PubMed PMC

69. Krupenko NI, Wagner C. Transport of rat liver glycine N-methyltransferase into rat liver nuclei. J Biol Chem 1997;272:27140-6. DOI PubMed

70. Wang YC, Lin WL, Lin YJ, Tang FY, Chen YM, Chiang EP. A novel role of the tumor suppressor GNMT in cellular defense against DNA damage. Int $J$ Cancer 2014;134:799-810. DOI PubMed

71. Raha A, Joyce T, Gusky S, Bresnick E. Glycine N-methyltransferase is a mediator of cytochrome P4501A1 gene expression. Arch Biochem Biophys 1995;322:395-404. DOI PubMed

72. Masson LF, Sharp L, Cotton SC, Little J. Cytochrome P-450 1A1 gene polymorphisms and risk of breast cancer: a HuGE review. Am J Epidemiol 2005;161:901-15. DOI PubMed

73. Ding B, Sun W, Han S, Cai Y, Ren M, Shen Y. Cytochrome P450 1A1 gene polymorphisms and cervical cancer risk: A systematic review and meta-analysis. Medicine (Baltimore) 2018;97:e0210. DOI PubMed PMC

74. Wei XP, Hu J. Cytochrome P450 1A1 exon 7 polymorphism and susceptibility to lung cancer in the Chinese population: an updated meta-analysis and review. Onco-Targets Ther 2015;8:1611-8. DOI PubMed PMC

75. Yu MW, Chiu YH, Yang SY, et al. Cytochrome P450 1A1 genetic polymorphisms and risk of hepatocellular carcinoma among chronic hepatitis B carriers. Br J Cancer 1999;80:598-603. DOI PubMed PMC

76. de la Vega M, Chapman E, Zhang DD. NRF2 and the Hallmarks of Cancer. Cancer Cell 2018;34:21-43. DOI PubMed PMC

77. Carvajal-Yepes M, Himmelsbach K, Schaedler S, et al. Hepatitis C virus impairs the induction of cytoprotective Nrf2 target genes by delocalization of small Maf proteins. J Biol Chem 2011;286:8941-51. DOI PubMed PMC

78. Yen $\mathrm{CH}$, Hsiao HH. NRF2 is one of the players involved in bone marrow mediated drug resistance in multiple myeloma. Int $J \mathrm{Mol} S \mathrm{Sci}$ 2018;19:3503. DOI PubMed PMC

79. Wu HC, Cheng MJ, Yen CH, Chen YMA, Chen IS, et al. Chemical constituents with GNMT-promoter-enhancing and NRF2-reduction activities from taiwan agarwood excoecaria formosana. Molecules 2020;25:1746. DOI PubMed PMC

80. Fernández-Ramos D, Fernández-Tussy P, Lopitz-Otsoa F, et al. MiR-873-5p acts as an epigenetic regulator in early stages of liver fibrosis and cirrhosis. Cell Death Dis 2018;9:958. DOI PubMed PMC

81. Fernández-Tussy P, Fernández-Ramos D, Lopitz-Otsoa F, et al. miR-873-5p targets mitochondrial GNMT-Complex II interface contributing to non-alcoholic fatty liver disease. Mol Metab 2019;29:40-54. DOI PubMed PMC

82. Kant R, Yen CH, Hung JH, et al. Induction of GNMT by 1,2,3,4,6-penta-O-galloyl-beta-D-glucopyranoside through proteasomeindependent MYC downregulation in hepatocellular carcinoma. Sci Rep 2019;9:1968. DOI PubMed PMC

83. Watson WH, Song Z, Kirpich IA, Deaciuc IV, Chen T, McClain CJ. Ethanol exposure modulates hepatic S-adenosylmethionine and Sadenosylhomocysteine levels in the isolated perfused rat liver through changes in the redox state of the NADH/NAD(+) system. Biochim Biophys Acta 2011;181:613-8. DOI PubMed PMC

84. Pascale RM, Simile MM, De Miglio MR, Feo F. Chemoprevention of hepatocarcinogenesis: S-adenosyl-L-methionine. Alcohol 2002;27:193-8. DOI PubMed

85. Sueblinvong V, Kerchberger VE, Saghafi R, Mills ST, Fan X, Guidot DM. Chronic alcohol ingestion primes the lung for bleomycininduced fibrosis in mice. Alcohol Clin Exp Res 2014;38:336-43. DOI PubMed PMC

86. Pascale R, Daino L, Garcea R, et al. Inhibition by ethanol of rat liver plasma membrane $(\mathrm{Na}+, \mathrm{K}+)$ ATPase: protective effect of Sadenosyl-L-methionine, L-methionine, and N-acetylcysteine. Toxicol Appl Pharmacol 1989;97:216-29. DOI PubMed 